\title{
Selección por conformación de reproductores de tilapia roja Oreochromis sp., mediante prueba de progenie
}

\author{
Selection by conformation of broodstocks of red tilapia \\ Oreochromis sp., by progeny testing
}

\begin{abstract}
Hermes Pineda S, ${ }^{1 *}$ M.Sc, Mónica Taborda A, ${ }^{2}$ Tec Agr, Alejandro Hernández $B,{ }^{2}$ Ing Agr.
${ }^{1}$ Politécnico Colombiano Jaime Isaza Cadavid, Facultad de Ciencias Agrarias, Medellín, Colombia. ${ }^{2}$ Profesional Independiente, Medellín, Colombia. *Correspondencia: hrpineda@elpoli.edu.co
\end{abstract}

Recibido: Noviembre de 2012; Aceptado: Febrero de 2013.

\section{RESUMEN}

Objetivo. Contribuir con la caracterización estadística y genotípica (media, media del error estándar, varianza y heredabilidad) de la Longitud Total (LT) y Longitud Altura del Lomo (LAL), y plantear una estrategia de selección para mejorar la calidad de la semilla de reproductores de tilapia roja Oreochromis sp. Materiales y métodos. El proyecto se realizó en la Granja Experimental y de Producción Piscícola (PCJIC), a un Km del municipio de San Jerónimo, distante $61 \mathrm{~km}$ al Occidente de Medellín (Antioquia). Se conformaron tres grupos de cruzamientos, cada uno en proporción de 3 hembras por 1 macho así: un cruce Hembras Blancas (HB) x Macho Rojo (MR) y dos cruces Hembras Blancas (HB) x Macho Blanco (MB). Las progenies fueron medidas a los 60 días post eclosión, teniendo en cuenta los supuestos estadísticos. Resultados. El ANOVA mostró que las progenies difieren significativamente $(p<0.001)$ para ambas variables continuas. La LT fue mayor para la progenie del cruzamiento HBXMB sin reversión $(8.04 \pm 1.04 \mathrm{~cm})$ y LAL fue mayor para la progenie HBXMR $(2.33 \pm 0.44 \mathrm{~cm})$. La correlación pareada entre las variables fue altamente significativa $(r \geq 0.79$, $\mathrm{p}<0.001)$. Las heredabilidades fueron 0.59 (LT) y $0.64(\mathrm{LAL})$. Los porcentajes de manchas en las progenies fueron HBxMR (34\%) y HBxMB no revertida (66\%). Conclusiones. La progenie del Grupo $1 \mathrm{HBxMR}$ tuvo los reproductores para una mejor selección por conformación, por lo que se sugiere aumentar el número de animales con mayor LAL. También, considerar la menor cantidad de manchas oscuras y realizar un manejo de reproductores por grupos independientes para selección masal.

Palabras clave: Heredabilidad, herencia, mejoramiento genético, morfometría, peces (Fuente: MeSH).

\section{ABSTRACT}

Objective. Contribute to the statistical and genotypic characterization (mean, standard error, variance and heritability) of the Total Length (LT) and Height (LAL), and suggest a selection strategy to improve seed quality in broodstock of red tilapia Oreochromis $s p$. Materials and methods. The project was conducted in the Experimental and Production Fish Farm (PCJIC), one Km from the town of San Jerónimo, $61 \mathrm{~km}$ to the west of Medellín (Antioquia). Three mating groups were formed, each 
Pineda - Selección por conformación de reproductores de tilapia roja Oreochromis sp. 3627

in proportion of 3 females and 1 male: a mating group of White Females (HB) $x$ Red Male (MR) and two mating groups White Females (HB) $\times$ White Male (MB). The offspring were measured at 60 days post hatching, taking into account statistical assumptions. Results. The ANOVA showed that offspring differ significantly $(p<0.001)$ for both continuous variables. The LT was greater for the offspring of HBXMB without reversion $(8.04 \pm 1.04 \mathrm{~cm})$ and $L A L$ was higher for the HBXMR offspring $(2.33 \pm 0.44 \mathrm{~cm})$. The paired correlation between variables was highly significant $(r \geq 0.79, p<0.001)$. Heritability were 0.59 (LT) and 0.64 (LAL). The dark spot percentages of the offspring were HBxMR (34\%) and nonreversed HBXMB (66\%). Conclusions. The progeny of Group 1 HBxHR had the better broodstocks for selection by conformation. It is suggested that the number of animals with greater LAL is increased. Additionally, it is important to consider the minor amount of dark spots in broodstock management by independent groups for mass selection.

Key words: Fish, genetic enhancement, heredity, heritability, morphometry (Source: MeSH).

\section{INTRODUCCIÓN}

La venta de alevinos está siendo afectada por la baja disponibilidad y calidad de la semilla, ocasionada por el mal manejo genético de un reducido grupo de reproductores en los sistemas de producción piscícolas (1), efectos que no han sido documentados suficientemente en las especies de cultivo nativas y foráneas en Colombia.

En el caso del híbrido de tilapia roja Oreochromis $\mathrm{sp}$, con el $76 \%$ de la producción en cautiverio, alrededor de $50.000 \mathrm{Kg} / \mathrm{año}$ (1), se procura mantener las características de buen color, sin manchas oscuras y lomo de mayor tamaño. El uso de un reducido grupo de animales como reproductores, trae como falencias: un aumento de la endogamia, semillas menos resistentes a los agentes patógenos, menor adaptabilidad a las condiciones ambientales y bajo rendimiento productivo en cautiverio debido a los cruzamientos entre reproductores de un mismo pedigrí (2).

Ciertamente en el caso de este híbrido, lo anterior está asociado al inadecuado manejo genético que se ofrece a los reproductores y a la restricción gubernamental de importación de especies foráneas, que permita aumentar la variabilidad genética en los cultivos. En consecuencia, los piscicultores han señalado una gran necesidad de aplicar técnicas en el mejoramiento genético de la semilla disponible, considerando los recursos técnicos y financieros mínimos existentes que les permitan alcanzar una mayor competitividad en el mediano y largo plazo (3).

El presente trabajo buscó determinar los elementos básicos para aplicar un método estadístico y genotípico en dos características de conformación, aplicables al buen manejo de los reproductores de tilapia roja Oreochromis sp, con el fin de obtener alevinos mejorados para la comercialización. De esta manera, se debe considerar la incertidumbre que proporcionan los reproductores de este híbrido y la respuesta que la interacción genético ambiental pueda establecer para cada granja piscícola en particular. Igualmente, proveer los lineamientos para un programa de mejora genética por selección direccional masal con un grupo reducido de animales.

\section{MATERIALES Y MÉTODOS}

Sitio de estudio. Este trabajo de tipo analítico, se realizó en la Granja Experimental y de Producción Piscícola John Jairo González Torres $\left(06^{\circ} 26^{\prime} 37.57^{\prime \prime} \mathrm{N}, 75^{\circ} 44^{\prime} 04.93^{\prime \prime} \mathrm{W}\right)$ en el municipio de San Jerónimo (Antioquia), situado a una altura de $780 \mathrm{msnm}$ y con temperatura ambiente de $27^{\circ} \mathrm{C}$. Se emplearon seis estanques de cemento de $5 \mathrm{~m}^{2}$ cada uno (tres para los reproductores y tres para sus progenies), con entrada y salida de agua independiente y aireación individual. El agua fue obtenida de la Quebrada Guaracú, y presentó los siguientes parámetros físico químicos: temperatura entre $23-25^{\circ} \mathrm{C}$, pH 7.5-8.0, dureza 120 ppm, oxígeno disuelto: $7.8 \mathrm{mg} / \mathrm{l}$, amonio $1.46 \mathrm{ppm}, \mathrm{Cl} 6.0 \mathrm{ppm}$ y alta turbidez en épocas de invierno (visibilidad menor a $20 \mathrm{~cm}$ ).

Muestras biológicas. Se seleccionaron 12 reproductores por su conformación, considerando las variables numéricas continuas de Longitud Total (LT) y Longitud Altura del Lomo (LAL), también conocida como Profundidad del Cuerpo (Figura 1). Asimismo, con menos manchas y sin malformaciones, como características fenotípicas externas. 
Volumen 18 Suplemento 2013

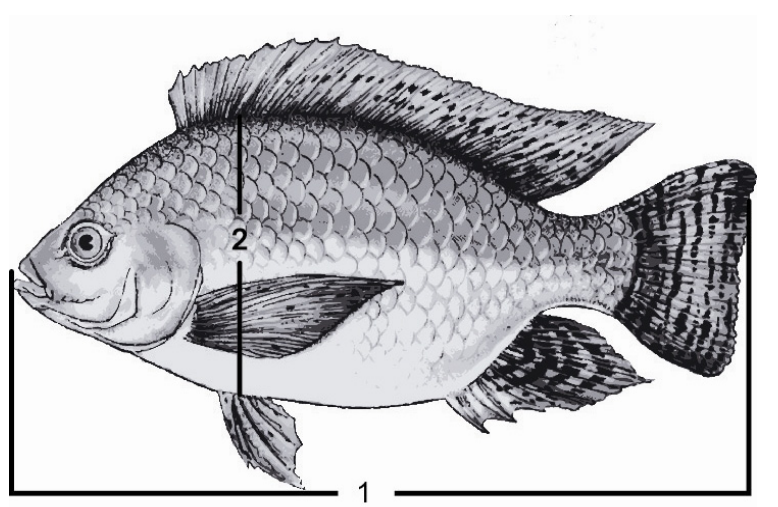

Figura 1. Mediciones morfométricas de tipo continuo consideradas en tilapia roja Oreochromis sp. 1. Longitud Total y 2. Longitud Altura del Lomo (Profundidad del Cuerpo). Fuente: PinedaSantis (4).

Se realizaron los siguientes cruzamientos:

Grupo 1: tres Hembras B (LT $\approx 41 \pm 1 ; \mathrm{LAL} \approx$ $11.7 \pm 0.2) x$ un Macho $R(L T=42.5 ; L A L=12.5)$. Grupo 2: tres Hembras B (LT $\approx 41 \pm 1$; LAL $\approx$ 11.7 \pm 0.2$) x$ un Macho $B(L T=41.5 ; L A L=12)$. Grupo 3: tres Hembras B $(\mathrm{LT} \approx 38.7 \pm 0.8 ; \mathrm{LAL} \approx$ $11.5 \pm 0.4) \times$ un Macho $B(L T=31.5 ; L A L=9.5)$.

Las progenies de los grupos 1 y 2 fueron alimentados con concentrado comercial no hormonado, con un alto porcentaje de proteína, mientras la progenie del grupo 3, fue alimentada durante un mes, con concentrado comercial (48\% de proteína), suplementado con la hormona 17-a-metiltestosterona para los procesos de masculinización fenotípica. El manejo de todas las progenies fue realizado por un único operario, manteniendo el mismo esquema general para la alimentación y las variables físico químicas del agua. De los tres grupos de reproductores se tomó, en promedio por progenie, entre 39 y 50 alevinos de ambos sexos, de los cuales se obtuvo la LT y la LAL a los 60 días post eclosión. Respecto al color de la piel en los reproductores, se estableció por comparación directa, un tono rojo menos intenso, algo rosado (más abundante en la granja piscícola), se denominó (B) y otro rojo más acentuado, se identificó como (R). No se conoce con certeza la causa de esta despigmentación, pero se apunta a las condiciones físico químicas del agua y al tiempo en cautiverio.

Aspectos éticos. Los reproductores tuvieron un manejo en campo, sujeto a las Buenas Prácticas Acuícolas. De esta manera, se consideraron las normas ambientales vigentes en la granja, relacionados con el uso de anestésicos y manejo general para un menor estrés de los animales, aspectos que fueron avalados por el Comité Pro témpore de la institución.

Análisis estadístico. Se seleccionaron alevinos en forma aleatoria de cada una de las tres progenies. Los datos obtenidos para cada una de las variables de respuesta, cumplieron con el supuesto de normalidad según Shapiro-Wilk. Se realizó la estadística descriptiva y el análisis de varianza $(p<0.05)$ entre las diferentes progenies con las comparaciones de TukeyKramer, las correlaciones pareadas de Pearson entre las variables y se estimó la heredabilidad mediante el uso del paquete estadístico $\mathrm{JMP}^{\circledR}$ y la aplicación Excel Microsoft ${ }^{\circledR}$. De la misma manera, se estableció el porcentaje de manchas en los animales para cada uno de los cruzamientos.

Los análisis de varianza para la estimación de la heredabilidad para la $L T$ y la $L A L$, se realizaron considerando los enunciados de Ruales et al (5), bajo los supuestos de aleatoriedad e independencia, normalidad (Prueba de ShapiroWilk) y homocedasticidad (Prueba de Bartlett). La partición entre grupos y dentro de grupos es la base para la estimación de la heredabilidad de la característica bajo estudio. Aquí se tiene en cuenta un supuesto adicional: la variación no genética es homogénea y se incluye en la variación dentro de grupos. Desde el punto de vista estadístico, el modelo de análisis fue:

$y_{i j}=\mu+G_{i}+\varepsilon_{i j}$ Donde,

$\mathrm{y}_{\mathrm{ij}}=$ valor de la característica en el individuo $\mathrm{j}$ $(j=1,2,3)$ del grupo genético $(G)$ i $(i=1,2,3), n_{i}$ siendo el número de individuos por grupo.

$\mu=$ promedio general de la característica

$\mathrm{G}_{\mathrm{i}}=$ efecto del grupo genético $\mathrm{i}$

$\varepsilon_{\mathrm{ij}}=$ error aleatorio asociado con el individuo $\mathrm{j}$ dentro del grupo i

Cálculo de heredabilidad $\left(\mathbf{h}^{2}\right)$. Desde el punto de vista de la variación, el modelo sería:

$\mathrm{VF}=\mathrm{V}_{\mathrm{A}}+\mathrm{V}_{\mathrm{NA}}+\mathrm{V}_{\mathrm{E}}$

Donde; $V_{F} V_{A}, V_{N A}, y V_{E}$ son las varianzas fenotípicas, aditivas, no aditivas y del entorno, respectivamente de la característica bajo estudio.

La heredabilidad en sentido estricto, se calculó como:

$$
h^{2}=\frac{V A}{V F}=\frac{V A}{V A+V N A+V E}
$$

Se utilizó el agrupamiento de hermanos completos ya que de un solo animal se generan muchos descendientes. Como el componente de varianza de hermanos completos, estima las varianzas aditivas y no aditivas, se dice 
Pineda - Selección por conformación de reproductores de tilapia roja Oreochromis sp. 3629

que el agrupamiento de medios hermanos es un estimador sesgado de la heredabilidad. Por lo tanto, la heredabilidad estimada por el agrupamiento de hermanos completos será:

$\mathrm{h}^{2}=\mathrm{V}_{\mathrm{A}} / \mathrm{V}_{\mathrm{F}}=2 * \sigma_{\mathrm{HC}}^{2} / \sigma_{\mathrm{HC}}^{2}+\sigma_{\mathrm{R}}^{2}$

Donde,

$\sigma_{\mathrm{HC}}^{2}=$ varianza de hermanos completos

$\sigma_{R}^{2}=$ varianza de reproductores

\section{RESULTADOS}

Las tres progenies presentaron diferencias significativas en la LT y la LAL ( $q=2.37 ; \mathrm{p}<0.001)$. El mayor promedio en la $\mathrm{LT}$, para animales con 60 días post eclosión fue registrado en la progenie HBXMB no revertida $(8.04 \pm 1.04 \mathrm{~cm})$ y el menor promedio fue para la progenie HBxMR $(6.02 \pm 0.99 \mathrm{~cm})$ (Tabla $1)$. Respecto a la $L A L$ fue mayor para la progenie HBXMR $(2.33 \pm 0.44 \mathrm{~cm})$ y menor para la progenie $\mathrm{HBXMB}$ revertida $(1.92 \pm 0.30 \mathrm{~cm})$, (Tabla 1). El Coeficiente de Variación no fue mayor a 30, asumiendo datos homogéneos, una confiabilidad en los datos y descartando los errores por azar. La normalidad se cumplió para los datos en todos los casos.

Tabla 1. Estadística descriptiva en tres progenies de tilapia roja Oreochromis sp con 60 días post eclosión.

\begin{tabular}{|c|c|c|c|c|}
\hline Parámetros & $\begin{array}{c}\text { Grupo } 1 \\
\text { Progenie } 1\end{array}$ & $\begin{array}{c}\text { Grupo } 2 \\
\text { Progenie } 2\end{array}$ & $\begin{array}{c}\text { Grupo } 3 \\
\text { Progenie } 3 \\
\text { Revertida }\end{array}$ & $\begin{array}{l}\text { ANOVA } \\
\text { (F) }\end{array}$ \\
\hline & \multicolumn{3}{|c|}{ Longitud Total } & \\
\hline Media \pm SEM $(\mathrm{cm})$ & $6.02 \pm 0.15^{\mathrm{a}}$ & $8.04 \pm 0.09^{b}$ & $6.63 \pm 0.25^{c}$ & $22.04 * * *$ \\
\hline $\mathrm{N}$ & 39 & 50 & 50 & \\
\hline \multirow[t]{2}{*}{$\mathrm{CV}$} & 16.4 & 13.0 & 9.8 & \\
\hline & \multicolumn{3}{|c|}{ Longitud Altura Lomo } & \\
\hline Media \pm SEM $(\mathrm{cm})$ & $2.33 \pm 0.04^{a}$ & $1.97 \pm 0.09^{b}$ & $1.92 \pm 0.05^{c}$ & $24.34 * * *$ \\
\hline $\mathrm{N}$ & 39 & 46 & 50 & \\
\hline $\mathrm{CV}$ & 18.8 & 14.6 & 15.6 & \\
\hline
\end{tabular}

$\mathrm{SEM}=$ Error estándar

Grupo 1: HBxMR; Grupo 2: HBxMB; Grupo 3: HBxMB.

$\mathrm{HB}=$ Hembra Blanca; $\mathrm{MR}=$ Macho Rojo; $\mathrm{MB}=$ Macho Blanco

$*(\mathrm{p}<0.05) ; * *(\mathrm{p}<0.01) ; * * *(\mathrm{p}<0.001)$

La correlación entre las variables fue altamente significativa $(r \geq 0.79, p<0.001)$, (Tabla 2$)$.

Tabla 2. Correlación pareada de dos características en la progenie de tilapia roja Oreochromis sp. con 60 días post eclosión.

\begin{tabular}{cccc}
\hline Parámetros & $\begin{array}{c}\text { Grupo 1 } \\
\text { Progenie 1 }\end{array}$ & $\begin{array}{c}\text { Grupo 2 } \\
\text { Progenie 2 }\end{array}$ & $\begin{array}{c}\text { Grupo 3 } \\
\text { Progenie 3 } \\
\text { Revertida }\end{array}$ \\
\hline LT $\times$ LAL & $0.79 * * *$ & $0.89 * * *$ & $0.88 * * *$ \\
\hline
\end{tabular}

$\mathrm{LT}=$ Longitud Total; LAL = Longitud Altura del Lomo Grupo 1: HBxMR; Grupo 2: HBxMB; Grupo 3: HBxMB. $\mathrm{HB}=$ Hembra Blanca; $\mathrm{MR}=$ Macho Rojo; $\mathrm{MB}=$ Macho Blanco.

$*(\mathrm{p}<0.05) ; * *(\mathrm{p}<0.01) ; * * *(\mathrm{p}<0.001)$
Con las varianzas se obtuvo los valores de heredabilidad de $\mathrm{LT}\left(\mathrm{h}^{2}=0.59\right)$ (Tabla 3) y $\mathrm{LAL}$ $\left(h^{2}=0.64\right)$ (Tabla 4).

Tabla 3. Análisis de varianza de la característica Longitud Total en las progenies de tilapia roja Oreochromis sp. con 60 días post eclosión.

\begin{tabular}{ccccc}
\hline $\begin{array}{c}\text { Fuente de } \\
\text { variación }\end{array}$ & gl & $\begin{array}{c}\text { suma de } \\
\text { cuadrados }\end{array}$ & $\begin{array}{c}\text { cuadrado } \\
\text { medio }\end{array}$ & $\begin{array}{c}\text { cuadrado } \\
\text { medio } \\
\text { esperado }\end{array}$ \\
\hline Entre padres & 2 & 69.634 & 34.82 & 0.66 \\
Dentro padres & 147 & 232.225 & 1.58 & 1.58 \\
\hline TOTAL & 149 & 301.859 & & \\
\hline
\end{tabular}

Heredabilidad $=2 *(0.66 /(0.66+1.58))=0.59$

Tabla 4. Análisis de varianza de la característica Longitud Altura del Lomo en las progenies de tilapia roja Oreochromis sp. con 60 días post eclosión.

\begin{tabular}{ccccc}
\hline $\begin{array}{c}\text { Fuente de } \\
\text { variación }\end{array}$ & gl & $\begin{array}{c}\text { suma de } \\
\text { cuadrados }\end{array}$ & $\begin{array}{c}\text { cuadrado } \\
\text { medio }\end{array}$ & $\begin{array}{c}\text { cuadrado } \\
\text { medio } \\
\text { esperado }\end{array}$ \\
\hline Entre padres & 2 & 10.362 & 5.18 & 0.10 \\
Dentro padres & 147 & 31.292 & 0.21 & 0.21 \\
\hline TOTAL & 149 & 41.654 & &
\end{tabular}

Heredabilidad $=2 *(0.10 /(0.10+0.21))=0.64$

Con relación al porcentaje de manchas, la progenie HBXMR fue de $34 \%$, más bajo que el porcentaje obtenido entre $\mathrm{HBXMB}$ no revertido $(66 \%)$.

\section{DISCUSIóN}

En cualquier sistema de producción animal se cruzan los mejores reproductores con el propósito de obtener progenies productivamente mejoradas, favoreciendo la frecuencia de genes con efecto aditivo, adaptados a las condiciones locales del estanque, haciendo el proceso de selección más eficiente $(6,7)$. A pesar de este principio, menos del $10 \%$ de la producción acuícola mundial están basados en programas de mejoramiento genético (8), por lo que se hace necesario potenciar este manejo con el fin de enfrentar la creciente demanda de proteína animal para una humanidad en expansión (9). Existen dos estrategias en donde la acción de los reproductores pueden cambiar las propiedades genéticas de la población: i. La selección de los animales que van a ser los padres y ii. El control de los cruzamientos, lo cual debe considerar la endogamia y la consanguinidad (10). En este caso, los resultados sugirieron que es posible seleccionar un grupo de reproductores de la granja piscícola para mejorar la calidad de la 
semilla en la primera progenie. Los animales del grupo 1 fueron los más pequeños en $L T$, pero sobresalen en la $L A L$, con una alta heredabilidad, sugiriendo un fuerte componente genético con lo cual se podrían realizar procesos de selección confiables para obtener un filete más grande. En este estudio no se consideró el color como criterio de selección, si bien, Garduno-Lugo et al (11) han sugerido una determinación homocigótica fijada para el color rojo mediante prueba de progenies en la quinta generación. Mather et al (12) sugirieron que el fenotipo rojo no afecta el crecimiento y puede ser mejorado por selección masal.

Las progenies de los Grupos 2 y 3 (HBxMB), presentaron diferencias en su conformación, debido al efecto genético y no al ambiental. Ciertamente, el proceso de inducción a la reversión sexual durante el primer mes de vida para la progenie del Grupo 3, moduló la maduración gonadal para los procesos metabólicos reproductivos sin incidir sobre la selección. Si bien los efectos epigenéticos no fueron evaluados en este trabajo, se ha considerado que los cíclidos, poseen una alta plasticidad para la adaptación a pequeños cambios en los nuevos ambientes, lo que les ha permitido su expansión en diversos ecosistemas acuáticos en todo el mundo $(13,14)$.

Al iniciar el funcionamiento de la granja piscícola, hace más de diez años, se recibieron las líneas Gregory Nielsen, Santafé, Huila, Repelón y Costain de tilapia roja Oreochromis $s p$, para conocer su adaptación a las condiciones de calidad de agua. Por desconocimiento del manejo en estas variedades, se perdieron las características de cada línea, aumentando la variación y la consanguinidad. Si bien es cierto, no se conoce el grado de afectación endogámica y se descuidó el manejo genético, las sucesivas generaciones de reproductores no han manifestado progenies con malformaciones evidentes. Aún así, la presencia de manchas oscuras en los animales ha sido considerada como un indicador del cruzamiento endogámico, por acción de su origen híbrido ontogénico, el cual fue evidenciado en animales con mayor tiempo en la granja (Grupo 2) (4).

Los reproductores viables para el mejoramiento genético y la obtención de alevinos mejorados, apuntarían a la selección de animales con mayor LAL, evidenciado en la progenie del grupo 1 . El reproductor masculino utilizado, una renovación de hace dos años, ofreció una mejor conformación. Doyle et al (6) consideraron el tamaño de los animales como característica apropiada para la selección, en lugar del peso o la edad, que siguen siendo los parámetros más valiosos en la producción $(15,16)$. No se descarta, en algunos casos, una selección indirecta que asocie el peso con parámetros de conformación.

Algunos trabajos han reportado menores heredabilidades en Longitud Total y en la Longitud Altura del Lomo para tilapia nilótica Oreochromis niloticus $(17,18)$, trucha arcoíris Oncorhynchus mykiss (19) y salmón Atlántico Salmo salar (20) que oscilan entre 0.25 y 0.46 . Por otra parte, existen otros reportes de altas heredabilidades para tilapia nilotica Oreochromis niloticus cultivados en tierra (21) y en jaulas (hapas) suspendidas en estanques fertilizados $\left(h^{2}>0.59\right)$ (22). Si bien los reportes han sido realizados para la tilapia nilotica Oreochromis niloticus, una especie válida y estable genéticamente, se considera que los resultados obtenidos para el híbrido de tilapia roja Oreochromis sp., cultivados en cemento, son confiables. Aún así, se deben continuar los procesos de trazabilidad para confirmar el desempeño durante la selección, lo anterior debido al escepticismo que predomina, sobre el manejo genético del híbrido.

En la granja piscícola es necesario aumentar el número de animales con mayor LAL que se encuentran en menor cantidad, y realizar cruzamientos con hembras de mayor LT, con menos manchas, para obtener progenies con un lomo más redondo o de mayor tamaño. Para lo anterior, se sugiere formar dos grupos independientes, entre 50 y 100 animales, que se encuentren en el promedio (a) y otros con promedio mayor (b) de la variable LAL. De lo anterior, se establece el diferencial de selección (S) (valor de los animales de promedio mayor (seleccionados) menos el valor promedio obtenido), es decir, $\mathrm{S}=\mathrm{b}-\mathrm{a}$. Es necesario que se obtengan los valores de las variables de los animales cada dos meses, para realizar su seguimiento y comparación con sucesivas progenies a la misma edad. Posteriormente, se obtendría la respuesta de la selección (R), el cual considera el valor promedio de la progenie obtenida (c), menos el promedio de los padres, a la misma edad $(\mathrm{R}=\mathrm{c}-\mathrm{a})$. Lo anterior, permitirá calcular la heredabilidad de la característica en sentido estricto $\left(h^{2}=R / S\right)$ (10). Este manejo, para pequeñas granjas con un reducido número de animales, fue sugerido por Doyle y Talbot (23), y probado por Brzeski y Doyle (24). Este manejo de animales, contribuiría a superar las falencias genéticas como endogamia a corto plazo, pérdida de variabilidad y reducción de frecuencias génicas asociadas a parámetros productivos, que siguen sin ser suficientemente documentados en las granjas piscícolas en Colombia. Por otro lado, 
Pineda - Selección por conformación de reproductores de tilapia roja Oreochromis sp. 3631

si el propósito es ajustarse a una selección genotípica, se hace necesario considerar la selección asistida con marcadores moleculares, los cuales permitirían mejores respuestas a la selección.

En conclusión la progenie obtenida del grupo 1 HBxMR, tuvo los reproductores para una mejor selección por conformación, por lo que se sugiere aumentar el número de animales con mayor LAL. Asimismo, considerar animales con menor cantidad de manchas oscuras y realizar un manejo de reproductores por grupos independientes para selección masal.

\section{Agradecimientos}

A la Dirección de Investigación y Posgrado por el apoyo financiero a este proyecto. A los directivos y al técnico profesional Jorge Rodríguez por su estimable colaboración, en la Granja Experimental y de Producción Piscícola John Jairo González Torres, en el municipio de San Jerónimo (Antioquia).

\section{REFERENCIAS}

1. Ministerio de Agricultura y Desarrollo Rural (MADR). Diagnóstico del estado de la acuicultura en Colombia: Plan nacional de desarrollo de la acuicultura sostenible en Colombia (FAO INCODER). Bogotá, Instituto Interamericano de Cooperación para la Agricultura, 2011.

2. Eknath $A E$, Doyle RW. Effective population size and rate of inbreeding in aquaculture of Indian major carps. Aquacult 1990; 85:293-305.

3. Ministerio de Agricultura y Desarrollo Rural (MADR). Agenda nacional de investigación en pesca y acuicultura. Bogotá, Instituto Interamericano de Cooperación para la Agricultura, 2012.

4. Pineda-Santis $H$, Arboleda-Chacón L. Caracterización morfométrica de tilapia roja Oreochromis sp para el mejoramiento genético en la estación piscícola del Politécnico en San Jerónimo (Antioquia). San Jerónimo-Antioquia: Politécnico Colombiano Jaime Isaza Cadavid. 2007.

5. Ruales FR, Manrique C, Cerón MF. Selección. En: Fundamentos en mejoramiento animal. Medellín, Vieco e hijas, 2007.

6. Doyle RW, Shackel NL, Basiao Z, Uraiwan S, Matricia T, Talbot AJ. Selective diversification of aquaculture stocks: a proposal for economically sustainable genetic conservation. Can J Fish Aquat Sci 1991; 48 Supl 1:148-154.

7. Bentsen HB, Olesen I. Designing aquaculture mass selection programs to avoid high inbreeding rates. Aquacult 2002; 204:349-359.
8. Gjedrem T. Genetic improvement for the development of efficient global aquaculture: a personal opinion review. Aquacult 2012; 344-349:12-22.

9. Gjedrem T, Robinson N, Rye M. The importance of selective breeding in aquaculture to meet future demands for animal protein: a review. Aquacult 2012; 350-353:117-129.

10. Falconer DS, Mackay T. Selection: I. the response and its selection. En: Introduction to quantitative genetics. 4th ed. London: Longman Group; 1996.

11. Garduno-Lugo M, Muñoz-Cordova G, Olvera-Novoa M. Mass selection for red colour in Oreochromis niloticus. Aquacult Res 2004; 35:340-344.

12. Mather PB, Lal SN, Wilson J. Experimental evaluation of mass selection to improve red body colour in Fijian hybrid tilapia (Oreochromis niloticus $\mathrm{x}$ Oreochromis mossambicus). Aqua Res 2001; 32:329-336.

13. Wagner CE, McIntyre PB, Buels KS, Gilbert $D M$, Michel E. Diet predicts intestine length in lake Tanganyika's cichlid fishes, Func Eco 2009; 23:1122-1131.

14. El-Sayed AFM. Basic biology and ecology. En: Tilapia culture. 2006. CAB Internactional. Oxfordshire (UK).

15. Gjedrem T. Selective breeding to improve aquaculture production. World Aquac 1997; 33-45. 
16. Charo-Karisa H, Komen J, Rezk MA, Ponzoni RW, Van Arendonk J, Bovenhuis $\mathrm{H}$. Heritability estimates and response to selection for growth of Nile tilapia Oreochromis niloticus in low-input earthen ponds. Aquacult 2006; 261:479-486.

17. Rutten MJM, Bovenhuis $H$, Komen $H$. Genetic parameters for fillet traits and body measurements in Nile tilapia Oreochromis niloticus. Aquacult 2005; 246:125-132.

18. Nguyen $\mathrm{NH}$, Khaw HL, Ponzoni RW, Hamzah A, Kamaruzzaman N. Can sexual dimorphism and body shape be altered in Nile tilapia Oreochromis niloticus by genetics means? Aquacult 2007; 272:28-46.

19. Gjerde B, Schaeffer LR. Body traits in rainbow trout: II. Estimates of heritabilities and of phenotypic and genetic correlations. Aquacult 1989; 80:25-44.

20. Powell J, White I, Guy D, Brotherstone S. Genetic parameters of production traits in Atlantic salmon Salmo salar. Aquacult 2008; 274:225-231.
21. Velasco RR, Janagap CC, De Vera MP, Afan LB, Reves RA, Eknath AE. Genetic improvement of farmed tilapias: estimation of heritability of body and carcass traits of Nile tilapia Oreochromis niloticus. Aquacult 1995; 137:280-281.

22. Charo-Karisa $H$, Bovenhuis $H$, Rezk MA, Ponzoni RW, Van Arendonk JAM, Komen $\mathrm{H}$. Phenotypic and genetic parameters for body measurements, reproductive traits and gut length of nile tilapia Oreochromis niloticus selected for growth in low-input earthen ponds. Aquacult 2007; 273:15-23.

23. Doyle RW, Talbot AJ. Effective population size and selection in variable aquaculture stocks. Aquacult 1986; 57:27-35.

24. Brzeski VJ, Doyle RW. A test of an on-farm selection procedure for tilapia growth in Indonesia. Aquacult 1995; 37:219-230. 